\title{
Database Design on Rotating Classroom Teaching Resources System based on DB2
}

\author{
Zuoyou Yin \\ College of Engineering, Bohai University, Jinzhou, 121013, China \\ yinzuoyou@163.com
}

Keywords: DB2; rotating classroom; teaching resources; management system; database design

\begin{abstract}
Rotating classroom teaching mode is based on abundant teaching resources. This paper is based on the DB2 database management system design. It can provide technical support for the development of teaching resource management system, and to carry out the rotation of classroom teaching mode of service. First, the conceptual structure design was carried out, and the E-R diagram was used to describe the conceptual model composed of five entities and their links; Then, the logical structure design is completed, the E-R model is transformed into the data structure model supported by the DB2 database management system; Finally, the index design is carried out. Five unique indexes and four non-unique indexes are designed according to the index design principle. In the design process, the paper fully considered the characteristics of rotating classroom teaching resources and DB2 database management system. It has the advantages of less data redundancy, clear structure and good integrity.
\end{abstract}

\section{Introduction}

Since Jon Bergmann and Aaron Sams put forward to "learn first to teach, teaching based on learning" as the core concept, flipped classroom teaching mode quickly become the focus of American university teaching reform which is through the network, micro-class and video resources and other technology-led. It also provides good experiences and references for the teaching reform in our country. The flipped classroom teaching mode is relying on information technology, students in the pre-class to complete the knowledge of learning, teachers to provide students with collaborative learning and exchange opportunities to help students achieve knowledge of internal learning. In this way affect students learning environment, so that students become the real master of learning. The flipped classroom teaching mode to re-adjust the time allocation within and outside the classroom. It will learn the decision to transfer from the teacher to the student, the core of the study is the students, teachers have become students to learn the designers, mentors, helpers and learning partners so that students and teachers to promote each other and common development. The reform of flipped classroom teaching model can reflect the advantages of "mixed learning", accord with the cognitive law of human beings, and help to construct new teacher-student relationship, which can promote the effective use of teaching resources [1,2].

Rotating classroom teaching mode is another kind of teaching mode which is developed on the basis of flipped classroom teaching mode. The study of the two processes were reversed, in the absorption of internalization of this link can be transferred from the students outside the classroom, in terms of learning effect and knowledge construction, with their own practical ability and foundation, consistent with the principle of individual teaching. The focus of the rotating classroom is to the autonomy of the classroom to the students, and teachers only through assistance and communication to assist students to learn, according to their own needs, students free to establish their own knowledge system. Rotating classroom teaching model is based on a wealth of teaching resources. In the information age, teaching resources are mainly stored in the database of digital resources, by the resource management system is responsible for unified management and maintenance, database design is the important work of developing resource management systems. Database design is the establishment of the database and its application system technology, which is an important part of software design. Specifically, for a given application environment, the optimal 
database schema is constructed, the database and its application systems are built to enable efficient storage of data to meet the needs of various users. This paper is based on DB2 database management system. It provides technical support for the development of teaching resources management system and services to the rotating classroom teaching.

\section{Product Components of DB2 Database}

IBM DB2 is a relational database management system developed by IBM in the United States. It is mainly used in large-scale application system, and has a good scalability, support from the mainframe to single-user environment, applied to all common server operating system platform. It provides high levels of data utilization, integrity, security and recoverability, as well as small scale to large-scale application execution capabilities, with platform-independent basic functions and SQL commands. With data grading technology, the mainframe data can be easily downloaded to the LAN database server, allowing client / server users and LAN-based applications to access mainframe data and transparentize database localization and remote connectivity [3]. It has a very complete query optimizer, external connections improve query performance, and support multi-task parallel query. The product components of the DB2 database are shown in Fig. 1.

\begin{tabular}{|c|c|c|c|c|}
\hline $\begin{array}{l}\text { DRDA } \\
\text { Application } \\
\text { Requester }\end{array}$ & DB2 Client & $\begin{array}{l}\text { RTCL: } \\
\text { Runtime } \\
\text { Client }\end{array}$ & & $\begin{array}{l}\text { DB2 Extender: } \\
\text { XML Extender } \\
\text { Net Search Extender } \\
\text { Spatial Extender } \\
\text { Geodetic Extender } \\
\text { Other additional } \\
\text { functional components }\end{array}$ \\
\hline$\downarrow$ & $\downarrow$ & $\downarrow$ & & $\downarrow$ \\
\hline \multicolumn{5}{|c|}{ Communication Support } \\
\hline \multirow[t]{3}{*}{ DB2 Engine } & & $\begin{array}{l}\text { Data Joiner } \\
\text { Relation Con } \\
\text { Web Sphere }\end{array}$ & & DB2 Connect \\
\hline & \multicolumn{2}{|c|}{$\nabla$} & & $\checkmark$ \\
\hline & \multicolumn{2}{|c|}{$\begin{array}{l}\text { Heterogeneous database: } \\
\text { Oracle, Informix, Sybase and } \\
\text { SQL Server }\end{array}$} & \multicolumn{2}{|c|}{$\begin{array}{l}\text { z/OS (large computer), } \\
\text { i/OS (medium-size computer) } \\
\text { DRDA Application Server }\end{array}$} \\
\hline
\end{tabular}

Fig. 1. Product components of DB2 database

DB2 Engine is the core of the entire database system and provides the basic functionality of DB2. The DB2 engine is similar to the car engine, responsible for the management and control of the data access; responsible for generating the package (storage access plan database object); to provide transaction management; to ensure data integrity and data protection; provide application concurrent control. The improvement of the database engine (DB2 Engine) determines whether the database system is stable and efficient. DB2 Engine is the most powerful database engine in all databases [4]. Communication support provides remote client support, client applications can access the database server through a variety of network protocols, TCP / IP, SNA (APPC / APPN), NETBIOS and Name Pipe and other protocols can be supported by DB2.

\section{Conceptual Structure Design}

The task of the conceptual structure design is to abstract the data model that is independent of any particular machine, that is, the conceptual model, according to the specific method based on the demand specification generated in the demand analysis phase. The conceptual model frees the designer's attention from complex implementation details and focuses only on the organizational 
structure and processing patterns of the most important information. The goal of the conceptual design is to accurately describe the information patterns in the application area, to support the user's various applications, both easy to convert to logical database model, and easy for the user to understand. The conceptual structure is the common ground of various data models, more independent than the data model, more abstract, and thus more stable. A powerful tool for describing conceptual models is the E-R model.

Rotating classroom teaching resources are complex, and the type of division is also difficult to unify. The system is first divided according to the curriculum, and then by resource type. Taking computer science and technology as an example, the course includes "data structure, programming language, operating system, computer composition principle, software engineering" and so on. "Resource types" include "text, images, audio, video, animation" and so on. The system consists of five entities, namely "curriculum, resource type, resource, resource file, resource evaluation". "Curriculum" and "resource type" is a one-to-many relationship, that is, a course of resources there are many types of resources; "Resource type" and "resource" are one-to-many links, that is, a "resource type" exists a variety of "resources", and a "resource" corresponds to only a "resource type"; "Resources" and "resource files" are one-to-many links, that is, a "resource" there are multiple "resource files", and a "resource file" only corresponds to a "resource"; "Resources" and "resource evaluation" are one-to-many links, that is, a "resource" there are multiple "resource evaluation", and a "resource evaluation" only corresponds to a "resource". The concept of the system structure design shown in Fig. 2.

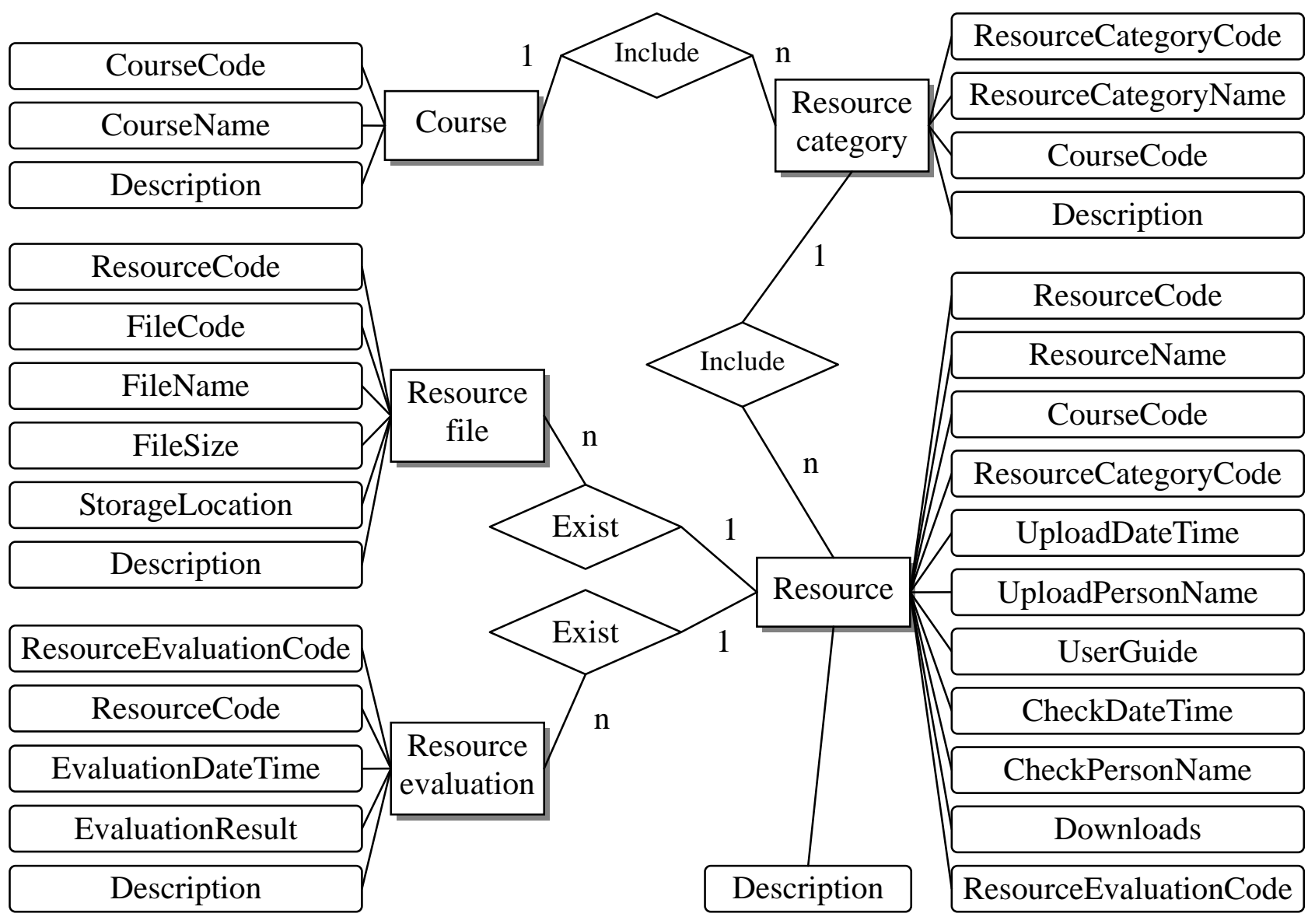

Fig. 2. E-R diagram on rotating classroom teaching resources system

\section{Logical Structure Design}

Logic refers to a collection of data elements that have one or more specific relationships. In order to establish the database required by the user, the conceptual model needs to be converted into a data model supported by a particular DBMS. The task is to convert the conceptual model into a data 
model supported by a particular DBMS, taking into account the characteristics of the specific data model and the performance of the DBMS. When an entity in the E-R diagram is converted to a relational schema, the attribute of the entity is the attribute of the relation. The code of the entity is the code of the relation. DB2 database includes a variety of data types, the system uses "char, varchar, date, int and clob" and other five types of data. The logical structure design results are shown in Table 1.

Table 1. Logical structure design on rotating classroom teaching resources system

\begin{tabular}{|c|c|c|c|c|c|c|c|}
\hline \multicolumn{4}{|c|}{ "Course" Table } & \multicolumn{4}{|c|}{ "Resource category" Table } \\
\hline No & Fields Name & Type & Bytes & No & Fields Name & Type & Bytes \\
\hline 1 & CourseCode & char & 10 & 31 & ResourceCategoryCode & char & 10 \\
\hline 2 & CourseName & varchar & 30 & 32 & ResourceCategoryName & varchar & 50 \\
\hline 3 & Description & clob & 1000 & 33 & CourseCode & char & 10 \\
\hline \multicolumn{4}{|c|}{ "Resource file" Table } & 34 & Description & clob & 1000 \\
\hline No & Fields Name & Type & Bytes & \multicolumn{4}{|c|}{ "Resource" Table } \\
\hline 11 & ResourceCode & varchar & 10 & No & Fields Name & Type & Bytes \\
\hline 12 & FileCode & varchar & 12 & 41 & ResourceCode & char & 10 \\
\hline 13 & FileName & varchar & 100 & 42 & ResourceName & varchar & 100 \\
\hline 14 & FileSize & decimal & 8,2 & 43 & CourseCode & char & 10 \\
\hline 15 & StorageLocation & clob & 1000 & 44 & ResourceCategoryCode & char & 10 \\
\hline 16 & Description & clob & 1000 & 45 & UploadDateTime & date & 7 \\
\hline \multicolumn{4}{|c|}{ "Resource evaluation" Table } & 46 & UploadPersonName & varchar & 30 \\
\hline No & Fields Name & Type & Bytes & 47 & UserGuide & clob & 2000 \\
\hline 21 & ResourceEvaluationCode & varchar & 15 & 48 & CheckDateTime & date & 7 \\
\hline 22 & ResourceCode & char & 10 & 49 & CheckPersonName & varchar & 30 \\
\hline 23 & EvaluationDateTime & date & 7 & 50 & Downloads & int & 4 \\
\hline 24 & EvaluationResult & clob & 1000 & 51 & ResourceEvaluationCode & varchar & 15 \\
\hline 25 & Description & clob & 1000 & 52 & Description & clob & 1000 \\
\hline
\end{tabular}

\section{Index Design}

DB2 index implementation is B + tree, which can achieve fast query, to avoid full table scan and reduce IO operation. Index is an abstract to the table data, by extracting the limited data, the data distribution is calculated, in order to complete the rapid retrieval of data. Create the index should follow the following principles [5,6]: The index includes all the columns in the "WHERE" clause, rejecting the unqualified rows in the result set by using the mask formed by the index; For indexes that are referenced in the "ORDER BY" clause of the SQL statement, indexes are created appropriately to avoid sorting; Taking into account the overhead of management, avoid using more than 5 columns in the index; For a multi-column index, place the most referenced column in the query; the larger base is suitable for creating the index; Do not include columns that are frequently modified or frequently inserted and deleted in the index. The only exceptions are primary keywords and foreign keywords; Can not be indexed with "null", any column that contains a "null" value is not included in the index, and any statement optimizer that uses "is null" or "is not null" in the "WHERE" clause is not used index; Use the "include" keyword to create an index. The system mainly creates two types of indexes:

(1) Unique index, used to ensure the uniqueness of the data.

The Course table creates a unique index in the CourseCode column with the name "IDX_Course_1".

The "Resource category" table creates a unique index with the name "IDX_ResourceCategory_1" in the "ResourceCategoryCode" column. 
The "Resource" table creates a unique index in the "CourseCode" column with the name "IDX_Resource_1".

The "Resource file" table creates a unique index in the "FileCode" column with the name "IDX_ResourceFile_1".

The "Resource evaluation" table creates a unique index with the name "IDX_ResourceEvaluation_1" in the "ResourceEvaluationCode" column.

(2) Non-unique index, used to improve the query speed.

The Course table creates a non-unique index in the CourseName column with the name "IDX_Course_2".

The "Resource category" table does not create a non-unique index because the amount of data in the table is small and the index is created instead of the query speed.

The "Resource" table creates a non-unique index in the "ResourceCode, ResourceName" column with the name "IDX_Resource_2".

The "Resource file" table creates a non-unique index with the name "IDX_ResourceFile_2" in the "ResourceCode, FileCode, FileName" column.

The "Resource evaluation" table creates a non-unique index with the name "IDX_ResourceEvaluation_2" in the "ResourceEvaluationCode, ResourceCode" column.

\section{References}

[1] X. Jin, X. J. Wang, "The implication and formation of the flipped classroom teaching culture," Educational theory and Practice, vol. 36, no. 5, pp. 7-9, 2016.

[2] Z. X. Liu, Q. H. Wu, "The cultivation of college students' autonomous learning ability from the perspective of flipped classroom," Modern educational technology, vol. 25, no. 11, pp. 67-72, 2015.

[3] S. Liu, "The Design and Optimization of DB2 Database," Information Security and Technology, vol. 2, no. 51, pp. 38-40, 2011.

[4] Red black alliance - IT technology learning website, "DB2 product components and functions," http://book.2cto.com/201307/27967.html, 2017-2-5.

[5] J. Q. Chen, "Performance tuning and optimization of DB2 database index," Master's degree of Shanghai Jiao Tong University, 2010.

[6] Y. J. Zhao, X. L. Chai, "B tree structure and example analysis of clustered index in database," Computer and Information Technology, vol. 24, no. 6, pp. 34-35, 2016. 